\title{
La ansiedad hacia la enseñanza de las matemáticas en estudiantes universitarios
}

\author{
Christofer José Canales López ${ }^{1}$ \\ Kenfor Misael Euceda Hernández ${ }^{2}$ \\ Luis Demetrio González Ponce ${ }^{3}$
}

\section{Información de artículo:}

\section{Recibido: 30/03/2021}

Aprobado:30/05/2021

Palabras claves:

Actitud, Ansiedad, Confianza, Conocimiento, Didáctica, Matemáticas.

\section{Resumen:}

En este artículo se definen las diferentes causas que propician que a un estudiante de matemáticas le cause ansiedad, entre los cuales existen factores como problemas sociales, relación de géneros y la modalidad de estudio, el objetivo es determinar la ansiedad de los estudiantes en la enseñanza de las matemáticas. En efecto, se pretende difundir los componentes de la ansiedad en el estudiante de la carrera de matemáticas, conociendo que las unidades temáticas no son flexibles para cualquier estudiante, eso induce al apasionado de la carrera a superar cualquier problema de ansiedad. También se analizan cada una de las variables y sus relaciones especificas para determinar cuales incrementan o reducen la ansiedad, esto a través de una encuesta tipo Likert, aplicada a 129 estudiantes de la Universidad Pedagógica Nacional Francisco Morazán de las sedes de Nacaome, Choluteca y Tegucigalpa de las modalidades de presencial y distancia, arrojando datos interesantes que podrían ayudar a mejorar los niveles de ansiedad.

\section{Anxiety towards the teaching of mathematics in students university}

\section{Abstract:}

This article defines the different causes that cause a math student to cause anxiety, among which there are factors such as social problems, gender relationship and modality of study, the goal is to determine students' anxiety in teaching mathematics. Indeed, it is intended to spread the components of anxiety in the student of the math career, knowing that thematic units are not flexible for any

\footnotetext{
${ }^{1}$ Profesor de Matemáticas en el Grado de Licenciatura, Docente del Instituto Técnico Contable John F. Kennedy ccanaleso61@ gmail.com, (1): https://orcid.org/0000-0002-8643-0612

2 Profesor de Educación Media en Matemáticas con orientación en Computación en el Grado de Licenciatura, Docente del Instituto Técnico Contable John F. Kennedy kenrakasth2009@gmail.com, (D): https://orcid.org/o0oo-0001-7340-9809

3 Profesor de Educación Media en Matemáticas con orientación en Física en el Grado de Licenciatura, Docente del Instituto Carlos Roberto Flores eldeme8o@gmail.com, (1): https://orcid.org/0000-0002-7400-822X
} 
student, that induces the passionate of the career to overcome any anxiety problem. Each of the variables and their specific relationships are also analyzed to determine which increase or reduce anxiety, this through a Likert-type survey, applied to 129 students from Francisco Morazán National Pedagogical University at Nacaome, Choluteca and Tegucigalpa headquarters of face-to-face and distance modalities, yielding interesting data that could help improve anxiety levels.

\section{Introducción}

El ambiente afectivo dentro de un entorno escolar es uno de los factores principales para que el estudiante perciba que el centro educativo es un lugar donde se aprende y se desaprende los conceptos erróneos, por tanto, se debe mostrar al estudiante que tan importante es el entorno que lo rodea, desafortunadamente vivimos realidades en donde los estudiantes se caracterizan por diversas formas, unos por aprender de forma rápida y otros por aprender de forma lenta. Quant y Sánchez (2012) afirman que "existen tipos de procrastinadores, sin embargo, señalan que en vez de siete tipos se pueden agrupar en dos: pasivos y activos" (Pardo, Perilla y Salinas 2014, p.33).

Decir que un estudiante se enfrenta a diversos problemas, es hacer énfasis en un aspecto que afecta en su aprendizaje, no obstante, existen estudiantes que se esfuerzan cada día por cumplir en realizar sus actividades de aprendizaje como aquellos que se conforman con realizar sus actividades a media. Por lo que este tipo de situación vuelve a un estudiante con rasgos de ansiedad frente a su aprendizaje, volviendo el proceso en algo no familiarizado con ellos. La ansiedad es un factor afectivo presente en los estudiantes, sobre todo en situaciones de evaluación o al enfrentarse a asignaturas especialmente difíciles para ellos, como pueden ser las matemáticas. Son numerosas las investigaciones que se han centrado en el estudio de la ansiedad hacia esta materia (Pérez-Tyteca et al., 2011, p.238).

Resultando oportuno entonces que la necesidad del estudio y las oportunidades que adquiere el estudiante da pauta a que caiga en el mundo de la ansiedad, causando en ellos diferentes cambios repentinos en su personalidad. De acuerdo con GuanoVillegas (2020) "los pensamientos automáticos son el cómo entendemos el contexto de los acontecimientos, la sensación que se generan y cómo reaccionamos siendo estos partes de los procesos cognitivos y afectivos" (p.6). Dentro del circulo de estudio encontraremos estudiantes de diversas características, pero mucho de ellos encuentran factores que les impide tener una empatía, esto debido a las peculiaridades de su proceso académico en años anteriores, querer encajar en una sociedad, es querer vivir complaciendo al que se tiene al lado.

El término inteligencia emocional, si bien fue utilizado por algunos autores previos, fueron Mayer y Salovey (1993), psicólogos de la Universidad de New Hampshire, quienes englobaron la inteligencia intrapersonal e interpersonal en el término inteligencia 
emocional. Con él, pretendieron describir las cualidades emocionales que parecen tener importancia para el éxito. Estas pueden incluir: la empatía, la expresión y comprensión de los sentimientos, el control de nuestro genio, la independencia, la capacidad de adaptación, la simpatía, la capacidad de resolver los problemas en forma interpersonal, la persistencia, la cordialidad, la amabilidad y el respeto (Otero-Martínez et al., 2009, p. 276).

\section{Literatura}

La ansiedad hacia la clase de matemáticas no es un tema nuevo hoy en día. Las matemáticas, por su nivel de abstracción, suelen ser difíciles de entender para la mayoría de las personas, especialmente para aquellas que no disfrutan dicho aprendizaje, ya sea porque simplemente no les gusta o por otras razones como ser no sentirse capaces de comprenderlas, sentirse muy inseguros, experiencias pasadas o incluso la personalidad del profesor. Sin embargo, no solo los estudiantes pueden manifestar ansiedad con respecto a la clase de matemáticas, también los docentes que la imparten. Las causas son variadas, en muchos casos, los maestros en formación desarrollan actitudes negativas hacia las matemáticas que son debidas a una débil base matemática, a sus experiencias previas con las matemáticas, la falta de apoyo de sus familias, y el efecto de sus clases de matemáticas anteriores (Tsao, 2014; Bolívar et al., 2014; Casis et al., 2017). Otros causales pueden ser los que señala Font (2011) y García-González y Martínez-Padrón (2020) las demandas de los sistemas educativos actuales generan malestar, preocupación y angustia en los docentes a quienes les cuesta concretar las herramientas adecuadas para organizar los contenidos matemáticos que deben enseñar, sobre todo cuando se les cuestiona la manera de enseñarlos en el aula, sumado a esto la muy poca colaboración de los estudiantes y la mala disciplina de estos.

En efecto, los múltiples factores que influyen a un estudiante son comprobado que la sobrecarga de actividades a realizar como responsables de estudio, y a la vez como embajadores del desarrollo de un país, conlleva a que esto tenga como resultados varios trastornos que afecte, logrando influir en sus estudios y esto más llevándolo consigo por vario tiempo a desempeñarlo frente a los estudiantes a un salón de clases o frente a la sociedad donde se encuentra. Debido a estas causas existen consecuencias que provoque efectos en los estudiantes, Veintimilla y Jamiled (2019) "efectos subjetivos, efectos conductuales, efectos cognoscitivos, efectos fisiológicos, efectos organizacionales" (p.11).

Es posible que la necesidad de obtener un adecuado rendimiento académico se pueda convertir en un factor estresante para los estudiantes. "Cuando la preocupación por tener una ejecución académica es alta, especialmente previa a los exámenes, la ejecución puede ser deficiente, al grado de que el alumno repruebe, postergue o abandone definitivamente sus estudios" (Riera-Murillo, 2020, p. 8). Siendo entonces la ansiedad causante de la disminución en el desempeño de sus tareas, reflejando un rendimiento 
académico no funcional, desarrollando malos hábitos, el cual estos podrían causar una permanencia en su estado de ánimo a largo plazo.

Mendías, Alex y Espigares (2011) afirman que entendemos que este rechazo hacia las matemáticas en los maestros en formación persistirá cuando ejerzan la profesión, convirtiéndose en una de las posibles causas a las que se puede atribuir el fracaso escolar, existen otros factores que pueden incidir en que se dé esta situación. Algunos de los más destacados son: la propia naturaleza de la materia, la metodología didáctica empleada durante las clases, las actitudes familiares y sociales y las características individuales de cada sujeto. Jadue (2001), el profesor capaz de percibir el sentimiento de impotencia en el alumno puede actuar para prevenir la ansiedad y facilitar el control. Los alumnos que presentan sentimientos de impotencia generalmente muestran hostilidad sistemática hacia un tema.

\section{Metodología de investigación}

\subsection{Finalidad y objetivos del estudio}

Esta investigación tiene por objetivo general determinar la ansiedad de los estudiantes en la enseñanza de las matemáticas, tomando en consideración las variables género, edad y grado académico de los estudiantes, por tales razones, se proponen los objetivos específicos siguientes: (1) Identificar la ansiedad del estudiantado en la enseñanza de las matemáticas; (2) Explicar la relación entre los factores actitudinales en la enseñanza de las matemáticas; (3) Comprobar si existen diferencias significativas entre género, edad y grado académico en relación con la ansiedad del estudiantado en la enseñanza de las matemáticas.

\subsection{Metodología de la investigación}

El enfoque de esta investigación es cuantitativo. Este paradigma a guiado el tratamiento de los datos a través de la categorización y descripción de las propiedades, características y perfiles de las personas, grupos, comunidades, procesos y objetos que se han sometidos a análisis (Hernández et al. 2014). El diseño de esta investigación es "Ex Post Facto" porque este tipo de investigación trata de descubrir fenómenos que ocurren en forma natural, pero miden diversas variables para analizar su posible efecto (Bisquerra, 2012).

\subsection{Descripción de los participantes}

El estudio se llevó a cabo con una muestra de 129 estudiantes de la Universidad Pedagógica Nacional Francisco Morazán de la Carrera de Matemáticas pertenecientes a comunidades del departamento de Valle, Choluteca y Francisco Morazán. 
Tabla 1. Participantes de la Investigación

\begin{tabular}{|l|c|c|c|}
\hline Grado Universitario (Carrera de Matemáticas) & Mujeres & Hombres & Total \\
\hline Primer Año & 2 & 2 & 4 \\
\hline Segundo Año & 10 & 14 & 24 \\
\hline Tercer Año & 15 & 9 & 24 \\
\hline Cuarto Año & 24 & 16 & 40 \\
\hline Ma de cuatro Años & 19 & 18 & 37 \\
\hline Total & 70 & 59 & 129 \\
\hline
\end{tabular}

El 54.3\% (70) de los participantes son mujeres, frente al 45.7\% (59) que son hombres. Respecto a su edad, la media se sitúa en 26 años, encontrándose al 37.2\% de los participantes en el intervalo de edad entre 23-28 años. En referencia a la variable grado académico universitario de la carrera de matemáticas: el $3.1 \%$ son del primer año ;18.8 \% son del segundo año; $18.8 \%$ son del tercer año; 31 \% del cuarto año; 28.9 \% son de más de cuatro años. Otra característica de la muestra es que la mayoría de los estudiantes utilizan tecnologías de la información y comunicación para acceder a sus estudios virtuales. Para concluir, hay que indicar que el tipo de muestreo que hemos utilizado ha sido el muestreo no probabilístico causal o accidental, que es aquel en el cual el investigador selecciona directa e intencionalmente la muestra, debido fundamentalmente a que tiene fácil acceso a la misma y es representativa de la población (Gil, Rodríguez \& García, 1995; Albert, 2006; Sabariego, 2004).

\subsection{Instrumentos de recogida de datos}

El instrumento para la recopilación de la información fue el cuestionario de ansiedad en la enseñanza de las matemáticas de Peker (2006), contextualizado por Gómez \& Caballero-Carrasco (2015). Según Gómez et al. (2015) el instrumento está constituido por 24 ítems, aglutinados en 4 dimensiones. Los estudiantes pueden indicar su agrado de acuerdo o desacuerdo con las afirmaciones propuestas por medio de una escala Likert de 5 valores. Los factores asociados son: conocimiento del contenido; confianza en sí mismo; actitud hacia la enseñanza de las matemáticas; y conocimiento didáctico. Es importante mencionar que la fiabilidad del instrumento fue un alfa de Cronbach del $96 \%$.

\subsection{Procedimiento de administración y enfoque ético}

La administración del instrumento se realizó por parte de los autores durante el primer semestre 2021. Tenía un carácter anónimo y fue complementado por los sujetos participantes en presencia del profesorado. Con anterioridad a la toma de datos, se obtuvo tanto el consentimiento previo, libre e informado de los participantes, así como la autorización de las autoridades educativas. 


\subsection{Análisis de datos}

Para satisfacer el objetivo del estudio se procedió al desarrollo de distintos análisis, empleando el paquete estadístico para las ciencias sociales SPSS v.25. Entre ellos, se aplicó un análisis de consistencia interna, un análisis descriptivo de los datos, un análisis de las correlaciones entre las categorías y pruebas paramétricas.

\section{Resultados}

\begin{tabular}{|c|c|c|c|c|c|c|}
\hline \multirow[b]{2}{*}{ Criterios } & \multicolumn{5}{|c|}{ Respuestas \% } & \multirow[b]{2}{*}{$\begin{array}{l}\text { Puntuación } \\
\text { Media (SD) }\end{array}$} \\
\hline & $\begin{array}{l}\text { Fuertemente } \\
\text { en desacu- } \\
\text { erdo }\end{array}$ & $\begin{array}{l}\text { En de- } \\
\text { sacu- } \\
\text { erdo }\end{array}$ & $\begin{array}{l}\text { Indife- } \\
\text { rente }\end{array}$ & $\begin{array}{l}\text { De } \\
\text { acu- } \\
\text { erdo }\end{array}$ & $\begin{array}{l}\text { Fuerte- } \\
\text { mente de } \\
\text { acuerdo }\end{array}$ & \\
\hline $\begin{array}{l}\text { 1. Siento que no se nada sobre los temas } \\
\text { de matemáticas que voy a enseñar. }\end{array}$ & 3.9 & 8.5 & 26.4 & 34.1 & 27.1 & $3.72(1.1)$ \\
\hline $\begin{array}{l}\text { 2. Temeré preguntar, sobre cuestiones } \\
\text { matemáticas que tendré que resolver, a } \\
\text { otros profesores. }\end{array}$ & 2.3 & 20.9 & 19.4 & 36.4 & 20.9 & $3.53(1.1)$ \\
\hline $\begin{array}{l}\text { 3. Pienso que será duro, difícil para mi re- } \\
\text { cordar las fórmulas matemáticas cuando } \\
\text { tenga que resolver cuestiones, en clase de } \\
\text { matemáticas. }\end{array}$ & 6.2 & 26.4 & 19.4 & 31.0 & 17.1 & $3.26(1.2)$ \\
\hline $\begin{array}{l}\text { 4. Pienso que me sentiré desesperado } \\
\text { cuando tenga que enseñar temas de } \\
\text { matemáticas }\end{array}$ & 4.7 & 13.2 & 17.1 & 37.2 & 27.9 & $3.71(1.1)$ \\
\hline $\begin{array}{l}\text { 5. Siento malestar (ansiedad) cuando se } \\
\text { menciona algunos temas de matemáticas } \\
\text { que debo enseñar. }\end{array}$ & 3.9 & 22.5 & 20.2 & 30.2 & 23.3 & $3.47(1.2)$ \\
\hline $\begin{array}{l}\text { 6. Temas matemáticos antes lo que sienten } \\
\text { ansiedad: divisiones, teorema de Pitágo- } \\
\text { ras, ecuaciones, raíz cuadrada, fracciones, } \\
\text { logaritmos, resolución de problemas, alge- } \\
\text { bra, derivada, integrales, geometría, funcio- } \\
\text { nes, límites, probabilidades, estadísticas, } \\
\text { aritméticas, formulas y matrices. }\end{array}$ & 5.4 & 18.6 & 27.9 & 28.7 & 19.4 & $3.38(1.2)$ \\
\hline $\begin{array}{l}\text { 7. Yo no tengo éxito para resolver prob- } \\
\text { lemas matemáticos. }\end{array}$ & 3.9 & 10.1 & 14.0 & 48.8 & 23.3 & $3.78(1.0)$ \\
\hline $\begin{array}{l}\text { 8. Tengo miedo de enseñar temas de } \\
\text { matemáticas. }\end{array}$ & 5.4 & 11.6 & 17.1 & 37.2 & 28.7 & $3.72(1.2)$ \\
\hline $\begin{array}{l}\text { 9. Sentiré malestar (ansiedad) cuando im- } \\
\text { parta clases de matemáticas. }\end{array}$ & 3.1 & 17.8 & 17.8 & 36.4 & 24.8 & $3.62(1.1)$ \\
\hline $\begin{array}{l}\text { 10. Es duro para mi enseñar la asignatura } \\
\text { de matemáticas. }\end{array}$ & 3.9 & 19.4 & 14.7 & 37.2 & 24.8 & $3.60(1.2)$ \\
\hline $\begin{array}{l}\text { 11. Pienso que será duro/difícil para mí } \\
\text { enseñar conceptos matemáticos. }\end{array}$ & 3.1 & 12.4 & 17.8 & 36.4 & 30.2 & $3.78(1.1)$ \\
\hline \multicolumn{7}{|l|}{ Puntuación Total ${ }^{a}$} \\
\hline Mediana (rango) & \multicolumn{6}{|l|}{$39(16-55)$} \\
\hline Media (SD) & \multicolumn{6}{|l|}{$39.55(9.90)$} \\
\hline
\end{tabular}


Tabla 2. Conocimiento del contenido

\begin{tabular}{|c|c|c|c|c|c|c|}
\hline \multirow[b]{2}{*}{ Criterios } & \multicolumn{5}{|c|}{ Respuestas \% } & \multirow[b]{2}{*}{$\begin{array}{l}\text { Puntuación } \\
\text { Media (SD) }\end{array}$} \\
\hline & $\begin{array}{c}\text { Fuertemente } \\
\text { en desacu- } \\
\text { erdo }\end{array}$ & $\begin{array}{l}\text { En de- } \\
\text { sacu- } \\
\text { erdo }\end{array}$ & $\begin{array}{l}\text { Indife- } \\
\text { rente }\end{array}$ & $\begin{array}{l}\text { De } \\
\text { acu- } \\
\text { erdo }\end{array}$ & $\begin{array}{l}\text { Fuerte- } \\
\text { mente de } \\
\text { acuerdo }\end{array}$ & \\
\hline Varianza & \multicolumn{6}{|l|}{98.10} \\
\hline Puntuación total \% & \multicolumn{6}{|l|}{$72 \%$} \\
\hline
\end{tabular}

El conocimiento de los contenidos en matemáticas es importante para cada docente, por tanto, debe ser igual para aquel futuro docente del área, por eso la dimensión de conocimiento del contenido se encuentra por arriba del valor medio, esto debido a que los futuros docentes consideran que es de suma importancia conocer cada unidad temática propuesta para los educandos, al hacer la relación del los criterios encontramos que aunque este sobre el valor medio, la pregunta 3 obtiene un 3.26 de la puntuación, queriéndonos decir esto que dentro de una salón de clases lo que debe permanecer en el docente es la seguridad de lo que va a impartir, de lo contrario podrá cometer errores por falta de conocimiento, resaltando el criterio 7 y 11 refleja una puntuación de 3.78, dando la pauta este puntaje para que el docente conozca que el éxito en la clase de matemática se obtiene si está preparado a enfrentar las situaciones didácticas que existen con los educandos, y a mismo tiempo las dificultades que conlleva el contenido, añadiendo entonces que es importancia conocer los contenidos para no encontrar dificultades al estar desarrollando la clase.

\begin{tabular}{|c|c|c|c|c|c|c|}
\hline \multirow[b]{2}{*}{ Criterios } & \multicolumn{5}{|c|}{ Respuestas \% } & \multirow[b]{2}{*}{$\begin{array}{l}\text { Puntuación } \\
\text { Media (SD) }\end{array}$} \\
\hline & $\begin{array}{l}\text { Fuerte- } \\
\text { mente en } \\
\text { desacu- } \\
\text { erdo }\end{array}$ & $\begin{array}{l}\text { En de- } \\
\text { sacu- } \\
\text { erdo }\end{array}$ & $\begin{array}{l}\text { Indife- } \\
\text { rente }\end{array}$ & $\begin{array}{l}\text { De acu- } \\
\text { erdo }\end{array}$ & $\begin{array}{l}\text { Fuerte- } \\
\text { mente de } \\
\text { acuerdo }\end{array}$ & \\
\hline $\begin{array}{l}\text { 12. Pienso que me sentiré relajado mientras } \\
\text { enseñe temas de matemáticas. }\end{array}$ & 4.7 & 15.51 & 19.4 & 41.1 & 19.4 & $3.55(1.11)$ \\
\hline $\begin{array}{l}\text { 13. Me siento capacitado como profesor para } \\
\text { resolver problemas matemáticos. }\end{array}$ & 0.8 & 13.2 & 26.4 & 39.5 & 20.2 & $3.65(0.97)$ \\
\hline $\begin{array}{l}\text { 14. Es muy fácil para mi enseñar temas de } \\
\text { matemáticas. }\end{array}$ & 3.9 & 21.7 & 30.2 & 31.8 & 12.4 & $3.27(1.06)$ \\
\hline $\begin{array}{l}\text { 15. Yo siempre tengo éxito para resolver } \\
\text { cuestiones matemáticas. }\end{array}$ & 1.6 & 19.4 & 33.31 & 34.1 & 11.6 & $3.35(0.97)$ \\
\hline $\begin{array}{l}\text { 16. Pienso que me sentiré relajado cuando } \\
\text { encuentre problemas matemáticos nuevos, } \\
\text { en clase como profesor. }\end{array}$ & 3.9 & 14.7 & 34.9 & 34.9 & 11.6 & $3.36(1.00)$ \\
\hline $\begin{array}{l}\text { 17. Pienso que siempre me sentiré relajado } \\
\text { en las lecciones de matemáticas. }\end{array}$ & 2.3 & 18.6 & 28.7 & 36.4 & 14.0 & $3.41(1.02)$ \\
\hline \multicolumn{7}{|l|}{ Puntuación Total a } \\
\hline Mediana (rango) & \multicolumn{6}{|l|}{$21(9-30)$} \\
\hline Media (SD) & \multicolumn{6}{|l|}{$20.59(5.27)$} \\
\hline
\end{tabular}




\begin{tabular}{|c|c|c|c|c|c|c|}
\hline \multirow[b]{2}{*}{ Criterios } & \multicolumn{5}{|c|}{ Respuestas \% } & \multirow[b]{2}{*}{$\begin{array}{l}\text { Puntuación } \\
\text { Media (SD) }\end{array}$} \\
\hline & $\begin{array}{l}\text { Fuerte- } \\
\text { mente en } \\
\text { desacu- } \\
\text { erdo }\end{array}$ & $\begin{array}{l}\text { En de- } \\
\text { sacu- } \\
\text { erdo }\end{array}$ & $\begin{array}{l}\text { Indife- } \\
\text { rente }\end{array}$ & $\begin{array}{l}\text { De acu- } \\
\text { erdo }\end{array}$ & $\begin{array}{l}\text { Fuerte- } \\
\text { mente de } \\
\text { acuerdo }\end{array}$ & \\
\hline Varianza & \multicolumn{6}{|l|}{27.73} \\
\hline Puntuación total \% & \multicolumn{6}{|l|}{$69 \%$} \\
\hline
\end{tabular}

a Máxima puntuación $=30$

Contradictoria respuestas en la dimensión de confianza en si mismo, a pesar de que no haya suficiente conocimiento, demuestran una confianza en lo que puedan impartir, observando que la puntuación media esta por encima del valor medio que es 3, destacando que el criterio 11 y 12 se sienten relajados al enseñar los temas de matemáticas y a laves capacitado para poderlos resolver. Añadiendo a eso los criterios 13,14,15,16, donde su mayor porcentaje esta en el ítem "de acuerdo" mencionando entonces que puede haber factores que intervienen el proceso de enseñanza aprendizaje, pero lo importante es tener confianza en lo que se vaya a realizar para demostrar que los estudiantes deben ver al profesor como un ejemplo y no como un obstáculo del conocimiento.

\begin{tabular}{|c|c|c|c|c|c|c|}
\hline \multirow[b]{2}{*}{ Criterios } & \multicolumn{5}{|c|}{ Respuestas \% } & \multirow[b]{2}{*}{$\begin{array}{l}\text { Puntuación } \\
\text { Media (SD) }\end{array}$} \\
\hline & $\begin{array}{l}\text { Fuerte- } \\
\text { mente en } \\
\text { desacu- } \\
\text { erdo }\end{array}$ & $\begin{array}{l}\text { En } \\
\text { desacu- } \\
\text { erdo }\end{array}$ & $\begin{array}{l}\text { Indife- } \\
\text { rente }\end{array}$ & $\begin{array}{l}\text { De acu- } \\
\text { erdo }\end{array}$ & $\begin{array}{l}\text { Fuerte- } \\
\text { mente } \\
\text { de acu- } \\
\text { erdo }\end{array}$ & \\
\hline $\begin{array}{l}\text { 18. Pienso que me gustará enseñar los con- } \\
\text { tenidos matemáticos. }\end{array}$ & 1.6 & 3.9 & 24.8 & 42.6 & 27.1 & $3.90(0.90)$ \\
\hline $\begin{array}{l}\text { 19. Pienso que será agradable para mí ense- } \\
\text { ñar los temas de matemáticas. }\end{array}$ & 1.6 & 3.1 & 23.3 & 48.1 & 24.0 & $3.90(0.86)$ \\
\hline $\begin{array}{l}\text { 20. Me gusta responder a las preguntas que } \\
\text { están relacionadas con los temas matemáti- } \\
\text { cos que se enseñan. }\end{array}$ & 1.6 & 7.8 & 27.1 & 41.1 & 22.5 & $3.75(0.94)$ \\
\hline $\begin{array}{l}\text { 21. Me gusta mostrar cómo se resuelven los } \\
\text { problemas matemáticos. }\end{array}$ & 3.1 & 5.4 & 20.2 & 48.1 & 23.3 & $3.83(0.95)$ \\
\hline \multicolumn{7}{|l|}{ Puntuación Total a } \\
\hline Mediana (rango) & \multicolumn{6}{|l|}{$16(4-20)$} \\
\hline Media (SD) & \multicolumn{6}{|l|}{$15.38(3.32)$} \\
\hline Varianza & \multicolumn{6}{|l|}{11.00} \\
\hline Puntuación total \% & \multicolumn{6}{|l|}{$77 \%$} \\
\hline
\end{tabular}

a Máxima puntuación $=20$

La tercera dimensión acerca de la actitud de la enseñanza de las matemáticas refleja una puntuación por encima del valor medio, por lo que se puede decir entonces que 
para impartir la clase de matemáticas siempre debemos pensar en lo que nos apasiona la clase, y así mismo reflejar en los estudiantes la misma actitud. Por tanto, el criterio 17 y 18 muestran una puntuación media de 3.90, el criterio 19 una puna puntuación de 3.75 y el criterio 20 de 3.83, entre el mas bajo de ellos cuatro está el criterio 19 que trata sobre responder a preguntas relacionadas con temas de matemáticas, ya que consideran que pueden llegar a confundir su aprendizaje.

\begin{tabular}{|c|c|c|c|c|c|c|}
\hline \multirow[b]{2}{*}{ Criterios } & \multicolumn{5}{|c|}{ Respuestas \% } & \multirow[b]{2}{*}{$\begin{array}{l}\text { Puntuación } \\
\text { Media (SD) }\end{array}$} \\
\hline & $\begin{array}{l}\text { Fuerte- } \\
\text { mente en } \\
\text { desacu- } \\
\text { erdo }\end{array}$ & $\begin{array}{l}\text { En de- } \\
\text { sacu- } \\
\text { erdo }\end{array}$ & $\begin{array}{l}\text { Indife- } \\
\text { rente }\end{array}$ & $\begin{array}{l}\text { De acu- } \\
\text { erdo }\end{array}$ & $\begin{array}{l}\text { Fuerte- } \\
\text { mente } \\
\text { de acu- } \\
\text { erdo }\end{array}$ & \\
\hline $\begin{array}{l}\text { 22. Creo que podría utilizar diferentes puntos } \\
\text { de vista y teorías sobre la enseñanza de las } \\
\text { matemáticas en mi vida docente. }\end{array}$ & 0.0 & 3.1 & 15.5 & 57.4 & 24.0 & $4.02(0.72)$ \\
\hline $\begin{array}{l}\text { 23. Podría usar modos de acceder al cono- } \\
\text { cimiento y métodos de investigación sobre la } \\
\text { enseñanza de las matemáticas, en mi vida } \\
\text { docente. }\end{array}$ & 0.0 & 3.1 & 11.6 & 62.8 & 22.5 & $4.05(0.68)$ \\
\hline $\begin{array}{l}\text { 24. Mientras enseñe matemáticas podría } \\
\text { usar información y estrategias relacionadas } \\
\text { con la educación especial. }\end{array}$ & 0.8 & 2.3 & 17.8 & 58.1 & 20.9 & $3.96(0.74)$ \\
\hline \multicolumn{7}{|l|}{ Puntuación Total a } \\
\hline Mediana (rango) & \multicolumn{6}{|l|}{$9(6-15)$} \\
\hline Media (SD) & \multicolumn{6}{|l|}{$12.03(1.86)$} \\
\hline Varianza & \multicolumn{6}{|l|}{3.47} \\
\hline Puntuación total \% & \multicolumn{6}{|l|}{$80 \%$} \\
\hline
\end{tabular}

En la dimensión 4 de conocimiento didáctico se refleja una superioridad, entre ellos está el criterio 22 estando por encima de la puntuación media con una puntuación de 4.05, destacando que se podrá usar modos de acceder al conocimiento y métodos de investigación sobre la enseñanza de las matemáticas, seguido del criterio 21 con una puntuación de 4.02, donde utilizar diferentes puntos de vistas y teorías sobre la enseñanza de las matemáticas en la vida docente y por último el criterio 23 con una puntuación media de 3.96 donde al enseñar matemáticas en necesario usar información y estrategias relacionadas con la educación especial. Entonces un porcentaje bastante alto reconoce que está en sus posibilidades investigar sobre nuevas teorías y técnicas de educación especial relacionadas con la enseñanza matemática, las cuales les servirán para mejorar su rol de docentes. Sin embargo, un pequeño porcentaje, entre el $3 \%$ y el $18 \%$ no está de acuerdo o no muestra interés en capacitarse para ser mejor docente de matemáticas, lo cual es preocupante pues el docente necesita estar en constante desarrollo. 
Tabla 6. Análisis de correlaciones entre las categorías ( $r$-Pearson)

\begin{tabular}{|l|c|c|c|c|}
\hline \multicolumn{1}{|c|}{ Factores } & Categoría 1 & Categoría 2 & Categoría 3 & Categoría 4 \\
\hline 1. Conocimiento del contenido & 1 & 0.739 & 0.688 & 0.517 \\
\hline 2. Confianza en sí mismo & & 1 & 0.720 & 0.527 \\
\hline $\begin{array}{l}\text { 3. Actitud hacia la enseñanza } \\
\text { de las matemáticas }\end{array}$ & & & 1 & 0.676 \\
\hline 4. Conocimiento didáctico & & & & 1 \\
\hline
\end{tabular}

Se observa entonces que los factores encontrados en el proceso de cada dimensión es directamente proporcional para un buen análisis de correlación, en efecto mantener un buen conocimiento y a la vez mejorar las debilidades genera una mejor confianza para que cada docente en matemáticas tenga una buena actitud y así dar paso un mejoramiento del conocimiento didáctico, esto ayudando a que cada vez nos interesemos en indagar en las nuevas estrategias que ayuden a mejorar esa calidad de enseñanza y así transmitir a los estudiantes el mejor conocimiento y a la vez mejorando las debilidades propias.

\begin{tabular}{|l|c|c|c|c|c|}
\hline \multicolumn{2}{|c|}{ Tabla 7: Análisis paramétrico o no paramétrico } \\
\hline \multirow{2}{*}{ Factores } & Género & Edad & $\begin{array}{c}\text { Año Aca- } \\
\text { démico }\end{array}$ & $\begin{array}{c}\text { Tipo de Es- } \\
\text { cuela }\end{array}$ & $\begin{array}{c}\text { Modalidad de } \\
\text { Estudio }\end{array}$ \\
\cline { 2 - 6 } & $p$ & $p$ & $p$ & $p$ & $P$ \\
\hline Conocimiento del contenido & 0.002 & 0.273 & 0.260 & 0.399 & 0.015 \\
\hline Confianza en sí mismo & 0.00 & 0.274 & 0.159 & 0.438 & 0.038 \\
\hline $\begin{array}{l}\text { Actitud hacia la enseñanza } \\
\text { de las matemáticas }\end{array}$ & 0.001 & 0.266 & 0.530 & 0.072 & 0.114 \\
\hline Conocimiento didáctico & 0.187 & 0.979 & 0.232 & 0.27 & 0.661 \\
\hline
\end{tabular}

Como se puede apreciar en la tabla precedente, el conocimiento de los contenidos, la confianza en sí mismo, la actitud hacia la enseñanza tiene diferencias significativas con respecto al género, no así el contenido didáctico. En cuanto a la edad, año académico y el tipo de escuela procedencia, no se observa diferencias significativas en relación con los factores. También se observan diferencias significativas en cuanto al conocimiento del contenido y confianza en sí mismo respecto a la modalidad de estudio.

\section{Discusión y conclusiones}

La ansiedad son comportamientos de inseguridad, desconfianza y preocupación de mujeres y hombres que exteriorizan como conductas, sentimientos y capacidades en el momento de sentir y pensar la solución de un problema matemático en contextos multiculturales (Flores-López, 2019), además, este factor se refiere al sentimiento de ansiedad, temor que el estudiante manifiesta ante la materia de matemática (FloresLópez \& Auzmendi, 2018). De los resultados obtenidos en el análisis de los datos se han extraído lo siguiente: 
La variable genero condiciona en cierta medida el conocimiento y la actitud hacia la enseñanza. Un porcentaje bastante alto, 27.9\%, afirma estar indeciso sobre si algunos temas de matemáticas les producen ansiedad, lo cual podría traducirse como inseguridad. Un $26.4 \%$ piensa que tiene problemas de memoria para recordar fórmulas matemáticas durante imparte la clase. La modalidad de estudio definitivamente influye en el nivel de conocimiento y en el grado de confianza que el futuro profesor pueda tener en sí mismo.

Respecto a la confianza en si mismo se destaca que un $41 \%$ del primer criterio en este factor, concederán sentirse relajados al momento de estar frente a sus estudiantes, demostrando la mejor confianza en ellos. No obviando los demás porcentajes ya que todos van inclinados a una mejor autoconfianza, siendo este primero el que más sobresale en su porcentaje. Conejeros, Rojas, Segure (2010) afirman que el sistema educacional es un importante agente socializador y promotor de confianza o desconfianza; la escuela no sólo entrega contenidos, sino que también opera en el mundo subjetivo de las relaciones interpersonales. Según se ha citado entonces el sistema educativo es el pilar fundamental para que cada educando refleje una actitud hacia la educación recibida, mostrando así patrones de comportamiento basado en la ansiedad. Además, Flores-López (2020) afirma que la confianza es un sentimiento de éxito y bienestar que provoca en mujeres y hombres el valor y capacidad de construir ideas para resolver problemas a partir del reconocimiento y familiarización del contexto educativo donde se desarrolle.

Como resultado de la actitud hacia la enseñanza de las matemáticas, se obtiene para el primer criterio un $42 \%$, que hace énfasis sobre el interés que se mostrara para dar la clase de matemáticas, $48 \%$ en relación a lo agradable que será dar cada tema, Posterior mente el $41 \%$ relacionado a la seguridad que mostrara cada docente al momento de responder preguntas que se relacionan con los temas de matemáticas, finalmente un $48 \%$ de los futuros docentes recomiendan mostrar a los estudiantes diversas formas de resolver cualquier ejercicios para aclarecer cualquier duda generada en ellos. FloresLópez y Auzmendi (2015) mencionan que los estudiantes con mejores actitudes hacia las matemáticas tienen más altas percepciones de la utilidad de las matemáticas, muestran motivaciones intrínsecas relacionadas con su estudio.

La relación encontrada en el conocimiento didáctico de los tres criterios, afirman que todos son importantes para una buena construcción de conocimiento, Considerando que es significativo utilizar diferentes puntos de vista para la enseñanza, y al mismo tiempo utilizar métodos que faciliten el acceso a los conocimientos didáctico, relacionando así cada estrategia. Resaltando entonces que el criterio 23 es que sobresale en su porcentaje, teniendo un $62 \%$ en su opción de acuerdo, por tanto, es necesario que cada docente se sienta comprometido al momento de dar su clase utilizando diversas formas de acceder a los contenidos, Acevedo (2009) define el conocimiento del contenido como "una combinación adecuada entre el conocimiento de la materia 
a enseñar y el conocimiento pedagógico y didáctico relativo a cómo enseñarla. El conocimiento del contenido en los futuros docentes es parte fundamental para que el educando obtenga el mejor aprendizaje por medio del facilitador, Nieto, Jiménez y Macias (1995), nos menciona que los profesores son los mediadores que transforman la materia en representaciones comprensibles a los alumnos, sabiendo entonces que es importante tener conocimiento de los contenidos.

Es importante señalar que el proceso de recolección de datos, muestra una gran relevancia en los resultados obtenidos, revelando lo sustancial que es llegar a tener un buen conocimiento matemático, y a la ves fijar las mejores actitudes frente a los estudiantes, considerando los diversos factores que intervienen en la enseñanza matemática, entonces para que el educando se sienta motivado, es necesario que el profesor se encuentre con la mejor actitud, contrarrestando todo aspecto negativo que lo influya por causa de la ansiedad vivida como aprendiz. Llinares (2013) menciona que una característica de los entornos de aprendizaje diseñados para fomentar el desarrollo de la competencia docente mirar profesionalmente la enseñanza-aprendizaje de las matemáticas es que se asume la relación dialéctica entre la construcción del conocimiento y el discurso.

\section{Los futuros docentes de matemáticas deben tomar en cuenta los siguientes as- pectos:}

1. Es importante saber que, el conocimiento de contenidos no solo se imparte de forma tradicional, sino que también mediante innovaciones atractiva para él futuro formador, construyendo asi ideas para poder transmitirlo con sus educandos y erradicar la típica frase de "no me gusta las matemáticas porque me dan dolor de cabeza" entonces si tomamos la decisión y actitud de formar a estudiantes, debemos tener en cuenta que elegimos transmitir saberes matemáticos, no miedo y conflictos.

2. Los docentes deben tener en cuenta que propiciar un excelente ambiente dentro del aula de clases, motiva a que el estudiante mejore su actitud y confianza para el desarrollo de los contenidos, El NTCM (2014) mencionan que "La enseñanza efectiva se basa, en parte, en una planificación cuidados. La co-planificación de una lección por parte de los docentes constituye una de las más grandes oportunidades de tener una influencia positiva en el aprendizaje del estudiante" (p.104).

3. El factor de la ansiedad involucra muchos elementos, y todo va a depender de la carga académica que el estudiante de matemáticas obtenga, entre mas presión educativa maneje será su nivel de ansiedad, entonces lo recomendado es que traten de realizar actividades extras que conlleve a un nivel de relajamiento mental. Para que se sienta funcional al momento de desarrollar las actividades. 
4. Comprender cada unidad temática, al desarrollar un estudio exhaustivo como educando, para que cuando se tenga la oportunidad de impartir el conocimiento con los estudiantes, no cause conflicto la comprensión de tema establecido en los libros de texto, ya que se carga mas el nivel de estrés en el docente, o lo que se puede llegar a interpretar en los estudiantes es inseguridad en la clase, por tanto, tener seguridad y preparación ayudaría a reducir la ansiedad en los estudiantes.

5. Las autoridades encargadas de regir y regular la educación de los futuros profesores de matemáticas deberían hacer énfasis en el manejo de los contenidos matemáticos por parte de los estudiantes, pues profesor que no maneja a cabalidad los contenidos que va a ensenar, sentirá miedo de equivocarse, y por lo tanto se sentirá ansioso al no saber si lo va a hacer bien frene a los alumnos.

6. El conocimiento didáctico es imprescindible en la formación de futuros profesores, pues no es lo mismo entender las matemáticas que saber enseñarlas, recordemos que la enseñanza de las matemáticas es toda una ciencia, por tanto, la didáctica general no es suficiente para que los docentes puedan transmitir de manera eficaz los contenidos matemáticos. Por lo tanto, los programas didácticos en el área de matemáticas deberían contar con procesos de formación más robustos orientados especialmente a la forma de enseñar matemáticas y más de acuerdo a la realidad nacional, no a situaciones idealizadas que pocas veces ocurren.

7. En ocasiones, los decentes de matemáticas titubean a la hora de dar sus clases, pues no se sienten lo suficientemente capacitados para enseñar bien determinado contenido. Este problema puede reducirse investigando mucho más a fondo sobre el tema en cuestión, no conformarse con lo que ha aprendido en sus estudios de grado, se pueden buscar nuevas maneras de resolver un mismo problema. El incremento de conocimiento y habilidades en el docente tendrá como resultado una mayor confianza y fluidez al momento de impartir las clases, pues es bien sabido que mejor maneje un tema un profesor, más fácil le resultara explicarlo a otros, en este caso, sus alumnos.

\section{Lista de referencias}

Acevedo, J. A. (2009). Conocimiento didáctico del contenido para la enseñanza de la naturaleza de la ciencia (I): el marco teórico. Revista Eureka sobre enseñanza y divulgación de las ciencias, 21-46. 
Albert-Gómez, M. J. (2006). La investigación educativa: Claves Teóricas. McGrawHill.https://www.academia.edu/download/59095661/LA_INVESTIGACION_ EDUCATIVA._CLAVES_TEORICAS20190430-128070-a4elay.PDF

Bisquerra-Alzina, R. (2012). Metodología de la investigación educativa. La Muralla.

Bolívar, D. P., Ballesteros, L. P., \& Ramírez, C. S. (2014). Relación entre procrastinación académica y ansiedad-rasgo en estudiantes de psicología. Cuadernos hispanoamericanos de Psicología, 14(1), 31-44.

Casis, M., Castro, N. R., \& Martínez, E. C. (2017). Motivación, autoconfianza y ansiedad como descriptores de la actitud hacia las matemáticas de los futuros profesores de educación básica de Chile. PNA, 11(3), 181-203.

Conejeros, M. L., Rojas, J., \& Segure, T. (2010). Confianza: un valor necesario y ausente en la educación chilena. Perfiles educativos, 32(129), 30-46.

Flores-López, W. O. (2019). Actitudes hacia las matemáticas en la resolución de problemas y su relación con la investigación propia. Managua: Editorial URACCAN. https:// bit.ly/3k3voyi

Flores-López, W. O. (2020). Significados sobre las actitudes hacia las matemáticas por estudiantes universitarios multiculturales. Revista Universitaria Del Caribe, 24(o1), 15-22. https://doi.org/https://doi.org/10.5377/ruc.v24io1.9907

Flores-López, W. O., \& Escribano, E. A. (2015). Análisis de la estructura factorial de una escala de actitud hacia las matemáticas. Aula de encuentro, 17(1).

Flores-López. W.O. \& Auzmendi, E. (2018). Actitudes hacia las matemáticas en la enseñanza universitaria y su relación con las variables género y etnia. Profesorado. Revista de Currículum y Formación de Profesorado, 22(3), 231-251. https://doi. org/10.30827/profesorado.v22i3.8000

Font, V. (2011). Investigación en didáctica de las matemáticas en la educación secundaria obligatoria. En M. Marín, G. García, L.J. Blanco y M. Medina (Eds.), Investigación en Educación Matemática XV, (pp. 165-194). Sociedad Española de Investigación en Educación Matemática y Servicio de publicaciones de la Universidad de Castilla-La Mancha

Gil, J., Rodríguez, G. y García, E. (1995). Estadística básica aplicada a las ciencias de la educación. Kronos. 
Gómez, R., \& Caballero-Carrasco, A. (2015). La ansiedad de los estudiantes para maestro ante la enseñanza de las matemáticas. En Blanco Nieto, L. J., Cárdenas Lizarazo, J. A., \& Caballero-Carrasco, A. (Eds.). La resolución de problemas de Matemáticas en la formación inicial de profesores de Primaria (59-80). Universidad de Extremadura.

González, M., \& Martínez-Padrón, O. (2020). Conocimiento emocional de profesores de matemáticas. Educación matemática, 32(1), 157-177.

Guano-Villegas, M. L. (2020). Distorsiones cognitivas y su repercusión en la ansiedad en un estudiante universitario en tiempos de pandemia (Bachelor's thesis, BABAHOYO: UTB, 2020).

Hernández-Sampieri, R., Fernández-Collado, C. y Baptista-Lucio, M. (2014). Metodología de la investigación. McGraw-Hill.

Jadue, G. (2001). Algunos efectos de la ansiedad en el rendimiento escolar. Estudios pedagógicos (Valdivia), (27), 111-118.

Llinares, S. (2013). El desarrollo de la competencia docente" mirar profesionalmente" la enseñanza-aprendizaje de las matemáticas. Educar em revista, (50), 117-133.

Mayer, J. D., \& Salovey, P. (1993). The intelligence of emotional intelligence.

Mendías, J. S., Alex, I. S., \& Espigares, A. M. (2011). Exploración de la ansiedad hacia las matemáticas en los futuros maestros de educación primaria. Profesorado. Revista de currículum y formación de profesorado, 15(3), 297-312.

National Council of Teachers of Mathematics (NCTM). (2014). De Los Principios A La Acción. Para Garantizar El Éxito Para Todos.

Nieto, L. B., Jiménez, V. M., \& Macías, C. R. (1995). Conocimiento didáctico del contenido en ciencias experimentales y matemáticas y formación de profesores. Rrubto de Educetodn, 607, 427-446.

Otero Martínez, C., Martín López, E., León del Barco, B., \& Vicente Castro, F. (2009). Inteligencia emocional y rendimiento académico en estudiantes de enseñanza secundaria. Diferencias de género.

Pardo, D., Perilla, L. y Salinas, C. (2014). Relación entre procrastinación académica y ansiedad - rasgo en estudiantes de psicología. Cuadernos Hispanomericanos de Psicología I, 14 (1), 31-44. Recuperado de: https://dialnet.unirioja.es/servlet/ articulo? codigo $=5493101$ 
Peker, M. (2006). Matematik Öğretmeye Yönelik Kaygi Ölçeğinin Geliştirilmesi. Journal of Educational Sciences \& Practices, 5(9), 73-92.

Pérez-Tyteca, P., Martínez, E. C., Romero, L. R., \& Martínez, E. C. (2011). Ansiedad matemática, género y ramas de conocimiento en alumnos universitarios. Enseñanza de las ciencias: Revista de investigación y experiencias didácticas, 237-250.

Quant, D. M., \& Sánchez, A. (2012). Procrastinación, procrastinación académica: concepto e implicaciones. Revista Vanguardia Psicológica Clínica Teórica y Práctica, 3(1), 45-59.

Riera-Murillo, M. L. (2020). Ansiedad y su influencia en el rendimiento académico de una estudiante (Bachelor's thesis, Babahoyo: UTB, 2020).

Sabariego, M. (2004). El proceso de investigación. En R. Bisquerra-Alzina, Metodología de la investigación edgucativa (127-163). La Muralla.

Tsao, Y. L. (2014). Attitudes and beliefs toward mathematics for elementary preservice teachers. US-China Education Review, 4(9), 616-626

Veintimilla, R., \& Jamiled, S. (2019). Factores estresantes y trastorno de ansiedad en un estudiante (Bachelor's thesis, BABAHOYO: UTB, 2019). 